\title{
Copeptin kinetics and brain edema/hemorrhagic transformation in acute ischemic stroke
}

Ornella Spagnolello*1 MD, Manuela De Michele ${ }^{2}$ MD PhD, Svetlana Lorenzano ${ }^{\star * 2}$ MD PhD, Emanuele Cerulli Irelli ${ }^{2}$ MD, Federico Naitana ${ }^{1}$ MD, Anne Falcou ${ }^{2}$ MD PhD, Federica Letteri ${ }^{2}$ MD, Alessandra Bachetoni ${ }^{3}$ MD, Daniela Collepardo ${ }^{4}$, Giuliano Bertazzoni ${ }^{1}$ MD, Danilo Toni ${ }^{2}$ MD PhD FESO

Emergency Department, Emergency Medicine, Policlinico Umberto I Hospital, Sapienza University, Rome, Italy, 2Department of Human Neurosciences, Stroke Unit, Policlinico Umberto I Hospital, Sapienza University, Rome, Italy, ${ }^{3}$ Department of Experimental Medicine, Clinical Pathology, Policlinico Umberto I Hospital, Sapienza University, Rome, Italy, ${ }^{4}$ Department of Surgery, Clinical Pathology, Sapienza University, Rome, Italy

Background and Purpose: Prognostic value of copeptin in acute ischemic stroke patients has been widely disclosed ${ }^{1,2}$. This study aimed to evaluate copeptin temporal profile according to reperfusion treatments and its potential relationship with the development of vasogenic brain edema and/or hemorrhagic transformation.

Methods: In this prospective cohort study, we performed serial assessment of copeptin and brain CT/MRI upon admission (TO), at 24 hours (T1) and within the third and the fifth day of hospitalization (T2) in 34 acute ischemic stroke patients. We evaluated copeptin kinetics along with the development of brain edema and/or hemorrhagic transformation, as well as functional outcomes and mortality at 3 months and 1 year (Table).

Results: Median copeptin concentration was 50,71 pmol/L at T0, $18,31 \mathrm{pmol} / \mathrm{L}$ at T1 and 10,92 pmol/L at T2. Copeptin assessed at T1 was higher in patients who had medium/severe brain edema at T2 (32.25 pmol/L medium/severe vs $13.67 \mathrm{pmol} / \mathrm{L}$ mild/absent edema; $p=0.038)$ and hemorrhagic transformation at T1 (93.10 $\mathrm{pmol} / \mathrm{L}$ vs $13.67 \mathrm{pmol} / \mathrm{L}: \mathrm{p}<0.003)$ and T2 (85.70 pmol/L vs 14.45 pmol/L: $p=0.024)$. Moreover, copeptin level drop (T0-T1) was steeper in patients who received reperfusion treatments, particularly in those undergoing i.v. thrombolysis plus mechanical thrombectomy (-129.34 pmol/L vs $-5.43 \mathrm{pmol} / \mathrm{L}$ combined therapy vs single reperfusion treatments: $p=0.038$ ) (figure). Drop in plasma copeptin levels also correlated with the $\mathrm{TICl}$ score of patients who underwent mechanical thrombectomy $(p<0001)$.

\begin{tabular}{|c|c|c|c|c|c|}
\hline & $\begin{array}{l}\text { All patients } \\
(\mathrm{N}=34)\end{array}$ & $\begin{array}{c}\text { Conservati } \\
\text { ve Therapy } \\
(\mathrm{N}=7)\end{array}$ & $\begin{array}{c}\text { Thromboly } \\
\text { sis alone } \\
(\mathrm{N}=13)\end{array}$ & $\begin{array}{c}\text { Thrombecto } \\
\text { my alone } \\
(\mathrm{N}=6)\end{array}$ & $\begin{array}{c}\text { Combined } \\
\text { Therapy } \\
(\mathrm{N}=8)\end{array}$ \\
\hline Age, years, mean & 75,5 & 69,3 & 75,3 & 62 & 69,7 \\
\hline Women, n (\%) & $16(47,1)$ & $3(42,8)$ & $8(61,5)$ & $1(16,7)$ & $4(50)$ \\
\hline $\begin{array}{r}\text { Copeptin T0, pmol/L, } \\
\text { median } \\
\end{array}$ & 50,71 & 31,18 & 26,71 & 60,71 & 174,15 \\
\hline $\begin{array}{l}\Delta \text { Copeptin (T0-T1), } \\
\text { pmol/L, median (IQR) }\end{array}$ & $\begin{array}{c}-11,28 \\
(-130.83 \\
5.90) \\
\end{array}$ & $\begin{array}{c}-2,70 \\
(-10.26 \\
25.90) \\
\end{array}$ & $\begin{array}{c}-4,50 \\
(-124.13 \\
18.53) \\
\end{array}$ & $\begin{array}{c}-37,84 \\
(-173.02 \\
5.68) \\
\end{array}$ & $\begin{array}{c}-129,34 \\
(-289.59,-28.01)\end{array}$ \\
\hline \multirow{3}{*}{\begin{tabular}{|} 
Post-infarct \\
Edema \\
COED 2-3, \\
n, (\%) T1
\end{tabular}} & $1 / 34(2,9)$ & $1 / 7(14,3)$ & 0 & 0 & 0 \\
\hline & $10 / 34(29,4)$ & $4 / 7(57,1)$ & $5 / 13(38,5)$ & 0 & $2 / 8(25)$ \\
\hline & $12 / 34(35,3)$ & $4 / 7(57,1)$ & $5 / 13(38,5)$ & $1 / 6(16,7)$ & $2 / 8(25)$ \\
\hline $\begin{array}{r}\text { Hemorragic } \\
\text { Transformation at T2, n, } \\
(\%) \\
\end{array}$ & $7 / 34(20,6)$ & $3 / 7(42,8)$ & $3 / 13(23,1)$ & 0 & $1 / 8(12,5)$ \\
\hline $\begin{array}{r}\text { mRS } 0-2(\%) \text { at } 1 \text { year, } \\
\text { n, (\%) }\end{array}$ & $18 / 33(54,6)$ & $3 / 7(42,8)$ & $8 / 13(61,5)$ & $4 / 6(66,7)$ & $3 / 7(42,8)$ \\
\hline $\begin{array}{r}\text { mRs 3-6 (\%) at } 1 \text { year, } \\
\text { n, (\%) }\end{array}$ & $8 / 33(24,2)$ & $2 / 7(28,6)$ & $2 / 13(15,4)$ & $2 / 6(33,3)$ & $2 / 7(28,6)$ \\
\hline Death at 1 year, $\mathrm{n},(\%)$ & $7 / 33(21,2)$ & $2 / 7(28,6)$ & $3 / 13(23,1)$ & 0 & $2 / 7(28,6)$ \\
\hline
\end{tabular}

Table. Clinical and neuroimaging features of acute ischemic stroke patients

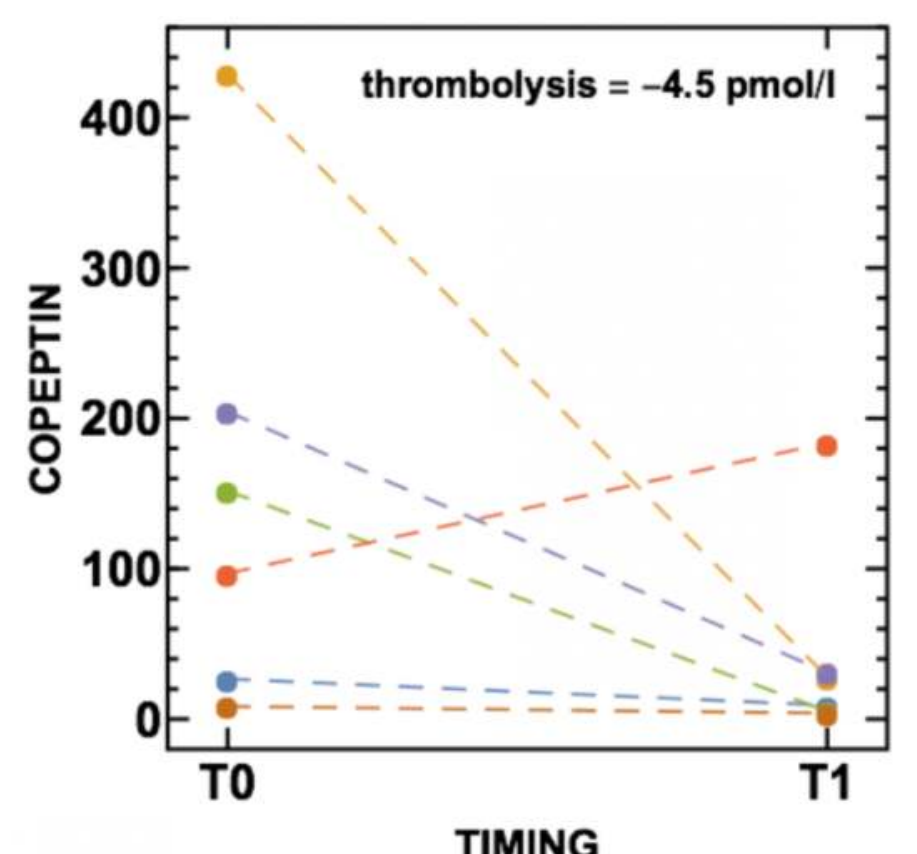

TIMING

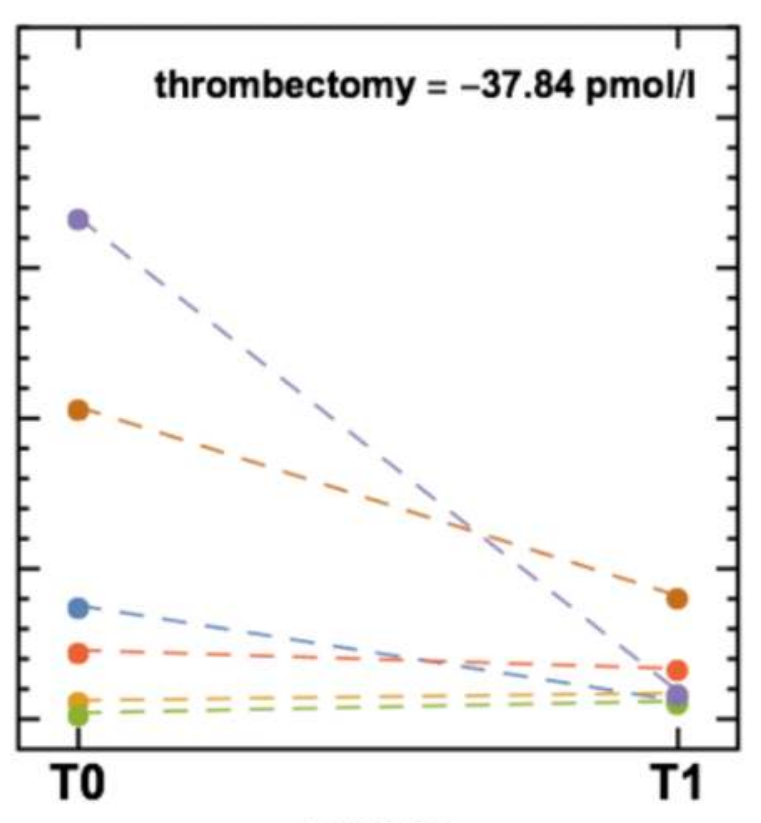

TIMING

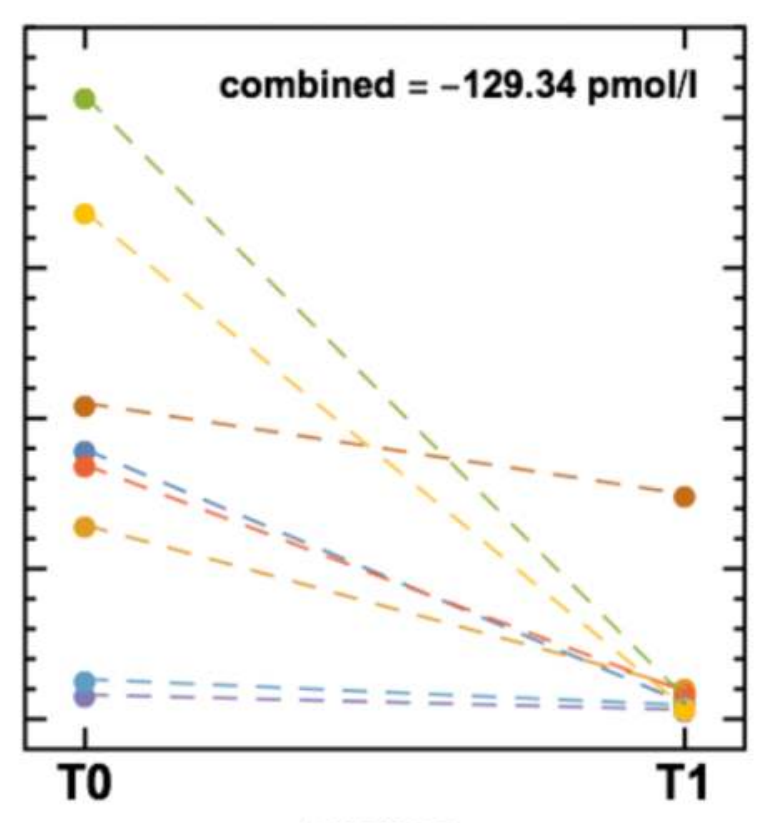

TIMING

Figure. The drop in copeptin levels (TO-T1) in treated patients, stratified by reperfusion treatment

Conclusions: Copeptin is a stress protein whose release in acute ischemic stroke seems to be strictly related to the development of brain edema and/or hemorrhagic transformation. It sharply drops after recanalization, mirroring the level of the achieved reperfusion in the acute phase of ischemic stroke. 